\title{
La nueva fórmula del trabajo: Revelaciones de Google que cambiarán su forma de vivir y liderar
}

\author{
Work Rules!: Insights from inside Google that will transform how you live and lead \\ Bettsy E. Mujica-Trejo ${ }^{a}$, Tirso J. Hernández-Gracia ${ }^{b}$, Karina Valencia-Sandoval ${ }^{c}$
}

\begin{abstract}
:
Google is already part of our lives, it has become the entity that knows everything and shows us all kinds of content in a few seconds. In the Human Resources department, the person in charge of this management and responsible for this is Laszlo Bock, who is vice president of personnel management and author of the book, the new formula of work, where he tells us about the non-traditional practices that have led to success in this area. It is essential that the work is motivating and human, since it is one of the biggest occupations in the life of the human being in society today. Among some valuable principles that are mentioned stresses that "pay unfairly, is the fairest". By continuing to read, it is also mentioned that most people believe that they spend huge amounts of dollars on various initiatives to facilitate the day to day of their employees, and invite these strategies can be copied to practice in other companies.
\end{abstract}

\section{Keywords:}

Human Resources, Google, employees, corporate culture, innovative strategies, leadership

\section{Resumen:}

Google ya es parte de nuestras vidas, se ha convertido en el ente que todo lo sabe ya que nos muestra todo tipo de contenidos en unos cuantos segundos. En el departamento de Recursos Humanos el encargado de esta gestión y responsable de ello es Laszlo Bock, quien es vicepresidente de gestión de personal y autor del libro la nueva fórmula del trabajo, donde nos narra las prácticas poco tradicionales que han llevado al éxito a esta empresa. Resulta primordial que el trabajo sea motivador y humano, ya que es una de las mayores ocupaciones de la vida del ser humano dentro de la sociedad actualmente. Dentro de algunos principios valiosos que se mencion an destaca que "pagar de forma injusta, es lo más justo". Al continuar con la lectura, también se hace mención de que la mayoría de las personas creemos que se gastan enormes cantidades de dólares en diversas iniciativas para facilitar el día a día de sus empleados, e invitan a que estas estrategias puedan ser copiadas para practicarse en otras empresas.

\section{Palabras Clave:}

Recursos Humanos, Google, Empleados, cultura corporativa, estrategias innovadoras, liderazgo.

\section{Introducción}

Google ya es parte de nuestras vidas, se ha convertido en el ente que todo lo sabe ya que nos muestra todo tipo de contenidos en unos cuantos segundos. Contratar a miles de personas en todo el mundo, es un proceso de expansión tan rápido que en esta empresa se siguen principios innovadores para hacer las cosas.

En el departamento de Recursos Humanos, el encargado de esta gestión y responsable de ello es Laszlo Bock, quien es vicepresidente de gestión de personal y autor del libro la nueva fórmula del trabajo, donde nos narra las prácticas poco tradicionales que han llevado al éxito a esta empresa. Este autor, menciona que se dedica a la gestión de personal porque quería cambiar la forma en que se les trata a los empleados.

Para Bock, resulta primordial que el trabajo sea motivador y humano ya que es una de las mayores ocupaciones de la vida del ser humano dentro de la sociedad actualmente. En el libro se menciona que, para conseguir miles de empleados llamados "googlers" sean más productivos y

\footnotetext{
a Autor de Correspondencia, Universidad Autónoma del Estado de Hidalgo, Instituto de Ciencias Económico Administrativas, Email: bemujicat@hotmail.com

${ }^{\mathrm{b}}$ Universidad Autónoma del Estado de Hidalgo, Instituto de Ciencias Económico Administrativas, Email: thernan@uaeh.edu.mx 
felices, se requiere de un manejo de datos y habilidades sociales.

\section{Principios y premisas importantes}

Ser capaz de contratar y persuadir a un trabajador que su trabajo no tiene por qué ser deprimente. Así mismo, al paso de la lectura, nos introduce en el interior de Google para explicar por qué es uno de los mejores lugares para trabajar y nos aporta principios sorprendentes que parecen sencillos y fáciles de implementar en cualquier empresa.

La nueva fórmula del trabajo parte de una premisa: "pasamos más tiempo trabajando que haciendo cualquier otra cosa. No resulta lógico que el trabajo sea una experiencia desmotivadora y poco humana". Esta idea, es el eje principal del libro, considero un paradigma a romper acerca de la capacidad para cambiar la forma en que trabajamos. Para Laszlo Bock, los empleados son el activo más valioso de cualquier empresa, perder a empleados con talento puede resultar realmente caro.

También, nos indica cómo podemos encontrar varios puntos de equilibrio entre creatividad y organización, así como un equilibrio entre el tiempo libre de los empleados y sus resultados; todo esto para conseguir un éxito conjunto, tanto para la empresa en cuestión, de generar valor y en la calidad de vida de sus "googlers".

Algunos principios valiosos que ofrece la lectura de este libro y que rompen la gestión convencional son:

1. Despoje de poder a los directivos y déselo a los empleados.

2. Aprenda de sus mejores personas, así como también de las peores.

3. Contrate solo a personas que sean más inteligentes que usted.

4. Pagar de forma injusta, es lo más justo.

A continuación expondré un poco estos principios:

El principio número 1 explica un poco el entorno, el autor del libro menciona que en Estados Unidos tienen jefes terribles y que en Google, cuando alguien adquiere un puesto que implica más responsabilidad, se le enseña a escuchar a los demás, a guiar al equipo a objetivos comunes. Laszlo Bock considera que los empleados deben tener mucho margen de decisión, más que sus propios jefes, esto ayuda a que los empleados se sientan más valorados.

El principio número 2 Google tiene un sistema de retribución que bonifica a los mejores empleados, de igual manera a los "no tan buenos" ya que se considera que todo es aprendizaje, también existe una prima para los fracasos que parten de ideas que la empresa valora positivamente.

El principio número 3 menciona que siempre se debe contratar a personas más inteligentes que uno mismo, aunque eso implique un mayor desembolso del capital.
El principio número 4 ha generado cierta controversia, ya que se considera que pagar de forma homogénea a los trabajadores es injusto, pagarles a los más sobresalientes de la empresa un $80 \%$ más, no es malo ni injusto, de hecho considera el autor que es lo correcto, ya que de no hacerlo el talento se puede ir a otra empresa.

Bock, pone a Google como ejemplo, pero argumenta que toda empresa puede implementar los mismos principios sin sacrificar los objetivos; como se puede leer en una de sus páginas y, que ciertamente coincido es: "cuando los empleados confían en sus líderes se convierten en embajadores de la empresa, y a su vez, fomentan un cambio progresivo en sus familias, la sociedad y el entorno".

Google como empresa ha implantado medidas innovadoras, del libro se pueden destacar algunas y sencillas de aplicar según la política de Recursos Humanos de Google que ellos Ilaman "People Operation" (Gestión de Personas), las 3 únicas formas de retener al talento, que a su vez crean un equilibrio en la organización son:

1. La calidad de las personas con las que se trabaja.

2. La sensación de que el trabajo que se realiza es significativo.

3. Mezclar la innovación, la eficiencia y el sentido de pertenencia.

En el país vecino, el autor del libro nos narra un poco de como las empresas gastan inclusive, hasta miles de millones en programas capacitación para sus empleados, dando a entender que aún si la formación que se da es buena, no hay manera de medir su impacto real en el trabajo, pues si no existe al final un cambio en la conducta de la persona, esa capacitación no es rentable.

El libro nos va presentando la forma en la Google fomenta la cultura dentro de la organización, así como la manera en que la mide y el hecho de que siempre busca tratar a las personas con decoro, explicando que esto se debe hacer y es la única forma en la que las demás empresas puedan tener un futuro en el área del conocimiento y en empresas que dependan del talento.

A continuación, se enlistan algunas de las cosas que los directivos en Google no pueden hacer, entre ellas destacan:

- A quién contratar.

- A quién despedir.

- Qué incremento salarial o acciones se otorgan.

- A quién se asciende.

- Cómo se evalúa el desempeño de algún empleado.

Esta empresa también tiene programas que permiten a los empleados disponer del $20 \%$ de su tiempo, lo que viene siendo equivalente a un día de trabajo por semana, para desarrollar proyectos personales, esta es una iniciativa del mismo Lazslo Bock, con la que él se mantiene firme en su 
filosofía, la cual es crear un ambiente de trabajo libre, creativo y lúdico.

Google fue pionera en incorporar elementos lúdicos en las oficinas, gracias a los fundadores de la compañía, a ellos se les ocurrió pensando en cómo sería la "oficina soñada", después se dieron a la tarea de poner comida gratis, piscina de bolas, restaurantes, etc. El objetivo es que los "googlers" hagan lo que deseen, en el momento que lo deseen.

Esta empresa en definitiva quiere hacer feliz a su personal, no controlar cuántas horas, ni el horario en que trabajan, donde trabajan, como trabajan, mientras más opciones para distraerse mejor y así fomenten la innovación, lo realmente importante es hacerlo bien.

Al continuar con la lectura, también se hace mención de que la mayoría de las personas creemos que se gastan enormes cantidades de dólares en diversas iniciativas para facilitar el día a día de sus empleados, e irónicamente se revela que muchos de sus programas son gratis y que son muy fáciles de copiar por otras empresas. Si es bien es cierto, la gente que se siente bien tratada produce mucho más; ellos lo demuestran al hacer tener este tipo de atenciones con sus empleados potenciando tres elementos básicos:

- Innovación

- Eficiencia

- $\quad$ Sentido de permanencia

Otra situación en la que se hace mucho énfasis, es en la forma de contratación, ya que menciona que es la parte más importante y se debe de hacer bien, de lo contrario terminaran al paso del tiempo preguntándose ¿por qué contraté a esa persona? Una mala contratación puede llegar a ser tóxica y enferma a todo el entorno de trabajo. Hay una cosa que es realmente única en Google en comparación con otras empresas y es que ésta utiliza datos científicos para poder medir todo lo que se hace en el departamento de Recursos Humanos. De acuerdo con el autor, Google utiliza internamente las herramientas que ofrece a sus clientes para medir los temas de cultura, analizar los datos estadísticos y temas relacionados con el capital humano, se manejan como experimentos o pruebas piloto.

El autor de este libro nos indica como las normas de Google, pueden funcionar para otras empresas que dependan del talento, sin importar si se dispone de mucho dinero de solo un poco, por otra parte, muchas de las ideas del vicepresidente de Recursos Humanos no son nuevas, muchas están inspiradas en Henry Ford.

Considero la parte más importante del libro está en el concepto de que si los Recursos Humanos son limitados, es necesario invertir en el área de capital humano, darse a la tarea de buscar a personas mejores, como ya se comentó en párrafos anteriores y que además, hacer de la contratación de personal un trabajo de todos en la empresa.

En la nueva fórmula del trabajo considera a la evaluación del desempeño como un desarrollo profesional y personal, cuyos objetivos están en función de cómo ser mejor y saber qué hacer, no en la etiqueta que produce la evaluación.

El hecho de que Google se ha dedicado a aprender y a emprender, no quiere decir que no hay cometido errores en el camino y todo haya funcionado a la primera, como una empresa que maneja la cultura innovadora, la experimentación y la tolerancia a las fallas, tienen una relevancia muy importante.

Bajo este contexto, el autor nos expone un ejemplo de ello, como se han recuperado y que es una aportación a esta reseña. Esta organización recompensa también económicamente el fracaso, ya que consideran que es fundamental y que de no hacerlo todos tendrían miedo de cometer errores y ya no se producirían ideas innovadoras y deja una lección valiosa: "se aprende más de un fracaso que de un éxito, porque el éxito no lo analizas y al fracaso sí".

Una anécdota que se cuenta en el libro es sobre Wayne Rosing, quien entonces fuera vicepresidente del departamento de ingeniería y que advirtió la salida de Google a la Bolsa a su equipo de trabajo, esto en 2004 les comentó que no quería encontrarse con un BMV en el estacionamiento y que de lo contrario lo quemaría.

Esto hizo que muchos empleados ganaran miles de dólares, cabe mencionar que, a los empleados de Google les pagan parte de su salario en acciones y lo que quiso decir Wayne Rosing, fue que en el ambiente de trabajo que se ha creado en esa empresa, la ostentación no es parte del entorno.; aunque por supuesto, en la actualidad se ven carros de ese tipo por los alrededores de la empresa.

Es un libro de lectura digerible y entretenido, revela secretos de una empresa exitosa y sin duda, con estas iniciativas y principios innovadores expuestos, Google es hoy en día, una de las empresas más importantes a nivel mundial y que año con año defiende su liderazgo.

Uno de los objetivos de Laszlo Bock al escribir este libro, es usar su experiencia al haber laborado en Google, poder responder ciertas interrogantes sobre el trabajo, por ejemplo: ¿Los equipos son mejores que las personas para hacer las cosas? ¿Pueden las personas mantener un alto rendimiento toda su vida?

\section{Referencias}

[1] Bock, L. (2015). La nueva fórmula del trabajo: Revelaciones de Google que cambiarán tu forma de vivir y liderar. Estados Unidos: Conecta. 